\title{
OPEN Systematic review with network meta-analysis of antivascular endothelial growth factor use in managing polypoidal choroidal vasculopathy
}

\author{
Sheng-Chu Chi ${ }^{1,7}$, Yi-No Kang ${ }^{2,3,4,5,7}$ \& Yi-Ming Huang ${ }^{1,6 \bowtie}$
}

Polypoidal choroidal vasculopathy (PCV) is a vision-threatening disease common in Asian populations. However, the optimal treatment for PCV remains under debate. We searched the databases with optimal searching strategy. The study included randomized clinical trials and prospective studies that recruited patients with active PCV who had received interventions, including PDT, anti-VEGF, or a combination of PDT and anti-VEGF. The Grading of Recommendations Assessment, Development, and Evaluation methodology was used for rating the quality of evidence. Our study included 11 studies involving 1277 patients. The network meta-analysis of RCTs revealed the anti-VEGF group, early combination group, and late combination group had significant BCVA changes compared with the PDT group. Early combination therapy led to a significant decrease in CRT compared with PDT, anti-VEGF, and late combination therapy. Additionally, the early combination group had a significantly higher complete polyp regression rate than the anti-VEGF group. No significant differences were detected in the analysis of the number of anti-VEGF injections and safety profile. This network meta-analysis revealed that early combination therapy exhibited better efficacy related to anatomical outcomes than other therapies. Nonetheless, no significant differences related to BCVA change could be detected between anti-VEGF and late combination therapy.

Polypoidal choroidal vasculopathy $(\mathrm{PCV})$ is considered a vision-threatening retinal disease. It is characterized by an abnormal inner choroidal branching vascular network with a nodular polypoidal aneurysmal lesion. The clinical features of PCV include reddish-orange nodular structures beneath the retina, serous pigment epithelial detachment, retinal pigment atrophy, and serous neurosensory detachment ${ }^{1}$. Antivascular endothelial growth factor (anti-VEGF) agents have been the first-line therapy for PCV because of their efficacy and safety ${ }^{2,3}$. Moreover, anti-VEGF can be combined with photodynamic therapy (PDT) for treating PCV 4 . PDT is another common treatment for PCV that targets the endothelial cells of vessels, resulting in the selective occlusion of polyp vessels and the resolution of active macular edema ${ }^{5}$. Notably, PDT can be used alone or in combination with anti-VEGF to treat $\mathrm{PCV}^{1,6}$. However, choosing the appropriate treatment for PCV is still a critical aspect of clinical practice.

Several prospective observational studies and randomized clinical trials (RCTs) have reported the efficacy of anti-VEGF agents, PDT, and their combination for treating $\mathrm{PCV}^{7-18}$. These studies have further subdivided combined therapy into early combination and late combination therapy. Notably, in early combination therapy, a patient receives PDT and anti-VEGF therapy at the beginning of the treatment course. However, in late combination therapy, patients receive anti-VEGF first, followed by rescue PDT.

Synthesized evidence from 4 studies was published before $2019^{19-22}$, and the latest meta-analysis derived conclusions from heterogeneous findings due to mixed data from retrospective studies and 2 RCTs. Notably,

\footnotetext{
${ }^{1}$ Department of Ophthalmology, Taiwan Faculty of Medicine, Taipei Veterans General Hospital, Taipei, Taiwan. ${ }^{2}$ Evidence-Based Medicine Center, Wan Fang Hospital, Taipei Medical University, Taipei, Taiwan. ${ }^{3}$ Research Center of Big Data and Meta-Analysis, Wan Fang Hospital, Taipei Medical University, Taipei, Taiwan. ${ }^{4}$ Cochrane Taiwan, Taipei Medical University, Taipei, Taiwan. ${ }^{5}$ Institute of Health Policy and Management, College of Public Health, National Taiwan University, Taipei, Taiwan. ${ }^{6}$ National Yang-Ming University School of Medicine, Taipei, Taiwan. ${ }^{7}$ These authors contributed equally: Sheng-Chu Chi andYi-No Kang. ${ }^{\square}$ email: nowaytokyo@yahoo.com.tw
} 
conceptual heterogeneity is unavoidable Consequently, the appropriate treatment strategy for PCV remains controversial.

Furthermore, several new RCTs were completed and published in 2017 and 2018 $8^{10,13,14}$. Therefore, an updated synthesis might confirm existing evidence and provide insight into PCV treatment. Hence, we conducted a systematic review with network meta-analysis to compare the effects of PDT monotherapy, anti-VEGF monotherapy, early combination therapy, and late combination therapy in treating PCV in terms of BCVA improvement, anatomical changes, and safety.

\section{Methods}

This systematic review with network meta-analysis of prospective studies was conducted to better understand the efficacy of PDT, anti-VEGF, and combination therapies in treating PCV; the study was designed in accordance with the Cochrane Handbook for Systematic Reviews of Interventions and registered on PROSPERO (CRD:42,020,181,736) beforehand. This synthesis was performed in accordance with the Preferred Reporting Items for Systematic Reviews and Meta-Analyses guidelines ${ }^{9}$. Institutional review board approval was not deemed necessary because the study used published data for analysis.

Eligibility criteria and evidence selection. Predefined eligibility criteria for evidence selection were as follows: (1) RCT or prospective study design; (2) study of patients with active PCV; and (3) use of interventions with PDT, anti-VEGF, or a combination of PDT and anti-VEGF. Exclusion criteria were as follows: (1) studies with unreported outcomes, for BCVA, proportion of patients with complete polyp regression, central retinal thickness (CRT) decrease, rates of adverse or ocular adverse events; (2) gray literature lacking a detailed report. On the basis of the different treatment modalities in the included trials, combination therapy was further subdivided into early combination and late combination therapy. Patients assigned to the late combination group where those who did not receive PDT initially but later received rescue PDT. Potential references were identified from the Cochrane Library, EMBASE, and New PubMed before February 2020. The search strategy consisted of using relevant terms such as "PCV," "PDT," and "anti-VEGF" in the free text and medical subject heading and using Boolean algebra. Details are provided in the Supplementary 1. Two reviewers (SCC and YNK) independently reviewed the references identified from the databases. Duplicates and irrelevant references were excluded through the screening of titles and abstracts after the search, and we retrieved full texts for further review of the remaining articles.

Data extraction and quality assessment. The 2 reviewers (SCC and YNK) independently identified and extracted relevant information, including study year; population characteristics; treatment modality; authors of studies; and outcomes, such as BCVA change, BCVA improvement rate, CRT decrease, the proportion of patients with complete polyp regression, number of anti-VEGF injections, adverse events, and ocular adverse events. The authors avoided double-count data from same trial or population by double checking relevant information of each trial. We used the Early Treatment Diabetic Retinopathy Study Visual Acuity Chart as the scale for BCVA. For continuous outcomes, mean and SD were extracted. If SE was presented in original trials, then $\mathrm{SD}$ was estimated from the sample size according to the relevant formula $(\mathrm{SE}=\mathrm{SD} / \sqrt{ } \mathrm{N})$. If relevant information of SD or SE could not be extracted from the original report, then authors were contacted. Imputation was employed using the maximum SD among eligible trials in the same outcome when the original SD could not be accessed. For instance, for the outcome of CRT decrease, Lee et al. and Koh et al. (2017) did not report relevant information for $\mathrm{SD}^{10,14}$, and imputation was performed. For binary outcomes, we extracted the event and total sample sizes.

With reference to relevant information regarding patient characteristics and outcomes, the 2 reviewers (SCC and YNK) independently assessed the bias of the included studies in the network meta-analysis by using the Risk of Bias 2 for RCTs and the Risk Of Bias in Non-randomized Studies of Interventions assessment. In addition, the Grading of Recommendations Assessment, Development, and Evaluation (GRADE) methodology was employed to rate the quality of evidence.

Data synthesis and analysis. This synthesis involved qualitative and quantitative analyses. All eligible studies were included in the qualitative synthesis, but only data from eligible RCTs were used in network metaanalysis to ensure high-quality evidence synthesis. All analyses were conducted using the random-effects model due to clinical heterogeneity. We reported continuous outcomes, in terms of weighted mean difference (WMD) and $95 \%$ CI. Dichotomous outcomes were reported using the risk ratio (RR) and 95\% CI. If an effect size raised clinical concerns without statistical significance, the surface under the cumulative ranking curve (SUCRA) was further determined to demonstrate the effects through hierarchical ranking of interventions.

Inconsistency and small-study effects were analyzed to evaluate the quality of network meta-analysis. Inconsistency analysis involved a loop inconsistency test according to Lu-Ades' method and the design-by-treatment interaction model. The primary method applied in the analysis of this synthesis was loop inconsistency, but the design-by-treatment interaction model was employed when an outcome was contributed by various arm designs. The adjusted funnel plot with Egger's test was employed for testing small-study effects. We assessed the statistical heterogeneity by using the $\mathrm{I}^{2}$ statistic.All analyses were performed using STATA version 14. 


\begin{tabular}{|c|c|c|c|c|c|c|c|c|c|c|c|c|c|}
\hline Study & $\begin{array}{l}\text { Study } \\
\text { type }\end{array}$ & Year & \begin{tabular}{|l|} 
Follow-up \\
time \\
(month)
\end{tabular} & Treatments & \begin{tabular}{|l|} 
Number \\
(eye)
\end{tabular} & $\begin{array}{l}\text { Age } \\
\text { (mean) }\end{array}$ & $\begin{array}{l}\text { Sex } \\
(M / F)\end{array}$ & $\begin{array}{l}\text { BCVA } \\
\text { (mean) }\end{array}$ & $\begin{array}{l}\text { CRT } \\
\text { (mean) }\end{array}$ & BCVAchange & CRTdecrease & $\begin{array}{l}\text { Completepolyp } \\
\text { Regression }\end{array}$ & Adverseevents \\
\hline $\begin{array}{l}\text { Chen } \\
\text { et al }\end{array}$ & $\begin{array}{l}\text { pro- } \\
\text { spec- } \\
\text { tive } \\
\text { cohort }\end{array}$ & 2018 & 12 & $\begin{array}{l}\text { Anti-VEGF } \\
\text { Combined }\end{array}$ & $\begin{array}{l}64 \text { whole } \\
\text { study }\end{array}$ & $\begin{array}{l}68.7 \\
\text { whole } \\
\text { study }\end{array}$ & $\begin{array}{l}41 / 23 \\
\text { whole } \\
\text { study }\end{array}$ & $\begin{array}{l}50 \\
\text { whole } \\
\text { study }\end{array}$ & $\begin{array}{l}310.8 \\
\text { whole } \\
\text { study }\end{array}$ & $\begin{array}{l}\text { Combined } \\
\text { superior to } \\
\text { Anti-VEGF }\end{array}$ & N/A & N/A & N/A \\
\hline $\begin{array}{l}\text { Chong } \\
\text { et al }\end{array}$ & $\begin{array}{l}\text { pro- } \\
\text { spec- } \\
\text { tive } \\
\text { cohort }\end{array}$ & 2018 & 12 & $\begin{array}{l}\text { ECT } \\
\text { LCT }\end{array}$ & $\begin{array}{l}41 \\
152\end{array}$ & $\begin{array}{l}70.7 \\
69\end{array}$ & $\begin{array}{l}18 / 41 \\
69 / 112\end{array}$ & $\begin{array}{l}46 \\
60\end{array}$ & N/A & - & N/A & N/A & N/A \\
\hline $\begin{array}{l}\text { Gomi } \\
\text { et al }\end{array}$ & RCT & 2015 & 12 & $\begin{array}{l}\text { ECT } \\
\text { LCT }\end{array}$ & \begin{tabular}{|c|}
37 \\
35
\end{tabular} & $\begin{array}{l}73.6 \\
73.8\end{array}$ & \begin{tabular}{|c|}
$37 / 0$ \\
$35 / 0$
\end{tabular} & $\begin{array}{l}54.3 \\
54.9\end{array}$ & $\begin{array}{l}360.5 \\
345.6\end{array}$ & - & - & - & - \\
\hline $\begin{array}{l}\text { Koh } \\
\text { et al }\end{array}$ & RCT & 2012 & 6 & $\begin{array}{l}\text { PDT } \\
\text { Anti-VEGF } \\
\text { ECT }\end{array}$ & \begin{tabular}{|l|}
21 \\
21 \\
19 \\
\end{tabular} & $\begin{array}{c}62.2 \\
69.3 \\
63.8 \\
\end{array}$ & \begin{tabular}{|l|}
$15 / 6$ \\
$15 / 6$ \\
$11 / 8$ \\
\end{tabular} & $\begin{array}{c}57.2 \\
49.0 \\
56.6 \\
\end{array}$ & $\begin{array}{l}285.3 \\
268.5 \\
334.7 \\
\end{array}$ & - & $\begin{array}{l}\text { ECT superior } \\
\text { to Anti- } \\
\text { VEGF } \\
\end{array}$ & $\begin{array}{l}\text { PDT, ECT superior } \\
\text { to Anti-VEGF }\end{array}$ & - \\
\hline $\begin{array}{l}\text { Koh } \\
\text { et al }\end{array}$ & RCT & 2017 & 24 & $\begin{array}{l}\text { Anti-VEGF } \\
\text { ECT }\end{array}$ & $\begin{array}{c}154 \\
168\end{array}$ & $\begin{array}{l}68.2 \\
68\end{array}$ & $\begin{array}{l}116 / 38 \\
109 / 59\end{array}$ & $\begin{array}{c}61.2 \\
61.1\end{array}$ & $\begin{array}{l}410.4 \\
415.9 \\
\text { (CSFT) }\end{array}$ & $\begin{array}{l}\text { ECT superior } \\
\text { to Anti- } \\
\text { VEGF }\end{array}$ & $\begin{array}{l}\text { ECT superior } \\
\text { to Anti- } \\
\text { VEGF }\end{array}$ & $\begin{array}{l}\text { ECT superior to } \\
\text { Anti-VEGF }\end{array}$ & - \\
\hline $\begin{array}{l}\text { Lai } \\
\text { et al }\end{array}$ & RCT & 2018 & 12 & $\begin{array}{l}\text { PDT } \\
\text { Anti-VEGF } \\
\text { ECT }\end{array}$ & $\begin{array}{c}23 \\
18 \\
19\end{array}$ & $\begin{array}{c}60.52 \\
64.67 \\
61.06\end{array}$ & $\begin{array}{l}14 / 9 \\
12 / 6 \\
10 / 6\end{array}$ & $\begin{array}{c}40 \\
32 \\
34\end{array}$ & $\begin{array}{c}478.04 \\
527.50 \\
522.63 \\
(\mathrm{CFT})\end{array}$ & - & - & $\begin{array}{l}\text { PDT superior to } \\
\text { Anti-VEGF }\end{array}$ & - \\
\hline $\begin{array}{l}\text { Lee } \\
\text { et al }\end{array}$ & RCT & 2018 & 13 & $\begin{array}{l}\text { ECT } \\
\text { LCT }\end{array}$ & $\begin{array}{l}161 \\
157\end{array}$ & $\begin{array}{l}70.4 \\
70.8\end{array}$ & $\begin{array}{l}112 / 49 \\
110 / 47\end{array}$ & $\begin{array}{l}59.0 \\
57.7\end{array}$ & $\begin{array}{c}346.1 \\
347.8\end{array}$ & - & - & - & - \\
\hline Li et al & $\begin{array}{l}\text { pro- } \\
\text { spec- } \\
\text { tive } \\
\text { cohort }\end{array}$ & 2018 & 12 & $\begin{array}{l}\text { Anti- VEGF } \\
\text { ECT }\end{array}$ & $\begin{array}{c}16 \\
48\end{array}$ & $\begin{array}{c}66.12 \\
68.44\end{array}$ & $\begin{array}{l}9 / 7 \\
28 / 20\end{array}$ & $\begin{array}{l}51.5 \\
50.05\end{array}$ & $\begin{array}{l}456.58 \\
467.64\end{array}$ & $\begin{array}{l}\text { ECT superior } \\
\text { to Anti- } \\
\text { VEGF }\end{array}$ & $\begin{array}{l}\text { ECT superior } \\
\text { to Anti- } \\
\text { VEGF }\end{array}$ & - & N/A \\
\hline $\begin{array}{l}\text { Lim } \\
\text { et al }\end{array}$ & RCT & 2012 & 12 & $\begin{array}{l}\text { Anti- VEGF } \\
\text { ECT }\end{array}$ & $\begin{array}{c}5 \\
5\end{array}$ & $\begin{array}{c}68.6 \\
57.8\end{array}$ & $\begin{array}{l}5 / 0 \\
3 / 2\end{array}$ & $\begin{array}{l}57.01 \\
50.99\end{array}$ & $\begin{array}{l}295.6 \\
213.4\end{array}$ & - & - & N/A & - \\
\hline $\begin{array}{l}\text { Oishi } \\
\text { et al }\end{array}$ & RCT & 2013 & 12 & $\begin{array}{l}\text { PDT } \\
\text { Anti- VEGF }\end{array}$ & $\begin{array}{l}47 \\
46\end{array}$ & $\begin{array}{c}75.0 \\
75.4\end{array}$ & $\begin{array}{l}32 / 15 \\
28 / 18\end{array}$ & $\begin{array}{l}56.5 \\
61\end{array}$ & $\begin{array}{l}366.8 \\
418.9\end{array}$ & $\begin{array}{l}\text { Anti-VEGF } \\
\text { superior to } \\
\text { PDT }\end{array}$ & & N/A & N/A- \\
\hline $\begin{array}{l}\text { Teo } \\
\text { et al }\end{array}$ & $\begin{array}{l}\text { pro- } \\
\text { spec- } \\
\text { tive } \\
\text { cohort }\end{array}$ & 2018 & 3 & $\begin{array}{l}\text { Anti- VEGF } \\
\text { ECT }\end{array}$ & $\begin{array}{c}13 \\
10\end{array}$ & $\begin{array}{l}68.7 \\
70.3\end{array}$ & $\begin{array}{c}7 / 6 \\
6 / 4\end{array}$ & $\begin{array}{c}67 \\
60\end{array}$ & $\begin{array}{l}352 \\
435.4\end{array}$ & - & - & $\begin{array}{l}\text { ECT superior to } \\
\text { Anti-VEGF }\end{array}$ & N/A- \\
\hline
\end{tabular}

Table 1. Characteristics of studies. -, No significant difference between two comparison; Anti-VEGF, antivascular endothelial growth factor; BCVA, best-corrected visual acuity; CRT, central retinal thickness; ECT, early combination therapy; LCT, late combination therapy; PDT, photodynamic therapy; N/A, no information of comparison in the study.

\section{Results}

Overall, 1036 potential articles were identified from the Cochrane Library $(\mathrm{k}=172)$, EMBASE $(\mathrm{k}=381)$, and New PubMed $(\mathrm{k}=483)$. After the removal of duplicates through systematic (EndNote) and manual matching, the remaining 747 articles were assessed. Finally, 4 prospective cohort studies ${ }^{12,15,16,18}$ and 7 RCTs $^{7-11,14}$ were included. The flow diagram is illustrated in Supplementary 2.

Characteristics and quality of included studies. Overall, 1277 patients were involved in the 11 studies (7 RCTs and 4 prospective cohort studies). Overall, there were 812 men. The available information revealed that the mean age of participants ranged from 61.98 to 73.70 years, and the baseline BCVA ranged from 38.5 to 63 letters. Some studies employed the central subfield thickness ${ }^{10,14}$ or central foveal thickness ${ }^{13}$ as scales to report the CRT. The baseline CRT among these participants ranged from 254.50 to 506.18 um. Details of the characteristics of the included studies are summarized in Table 1. The anti-VEGF agents, PDT protocol, rescue therapy, and criteria of rescue therapy are listed in Table 2. Most of the trials used ranibizumab as the anti-VEGF agent. Regarding PDT protocol, most trials employed standard-fluence PDT. The results of the study appraisal are summarized in Supplementary 3-4. The network plot of primary outcome was illustrated in Fig. 1, and forest plots of other main finding was presented ion Fig. 2. Summary of other outcomes was showed in Table 3. No asymmetry was noted upon visual inspection of all funnel plots (Supplementary 5-11) No inconsistency or small-study bias was noted in analysis of all outcomes. (Supplementary 12-18) Furthermore, SUCRA value was demonstrated in BCVA change, BCVA improvement rate and number of anti-VEGF needed. (Supplementary 19-21).

Efficacy. BCVA change. Regarding the outcome of BCVA change, a total of 7 RCTs revealed this outcome $^{7-10,13,14,17}$. In the consistency model, the results revealed significant differences in BCVA change between the anti-VEGF group and PDT group (WMD: 3.22; 95\% CI: $0.68,5.76$ ), the early combination group and PDT group (WMD: 4.59; 95\% CI: 1.95, 7.22), and the late combination group and PDT group (WMD: 4.67; 95\% CI: $1.68,7.67)$. No significant differences were detected among anti-VEGF, early combination, and late combination groups. Among the prospective cohort studies, all 4 studies reported the data of BCVA change. Teo et al. and 


\begin{tabular}{|c|c|c|c|c|}
\hline Study & $\begin{array}{l}\text { Anti-VEGF } \\
\text { treatment }\end{array}$ & PDT protocol & Rescue therapy & Rescue criteria \\
\hline Chen et al & $\begin{array}{l}\text { Ranbizumab } \\
0.5 \mathrm{mg} \text { according } \\
\text { to local practice in } \\
\text { Taiwan }\end{array}$ & N/A & $\begin{array}{l}\text { 1. Anti-VEGF group: PRN additional } \\
\text { anti-VEGF according to local practice } \\
\text { in Taiwan } \\
\text { 2. Combined group: PRN additional } \\
\text { anti-VEGF and PRN PDT according } \\
\text { to local practice in Taiwan }\end{array}$ & According to local practice in Taiwan \\
\hline Chong et al & \begin{tabular}{|l|} 
Monthly \\
bevacizumab, \\
ranbizumab, or \\
aflibercept \\
According to real \\
world practice \\
\end{tabular} & N/A & $\begin{array}{l}\text { 1. Early combination group: PRN } \\
\text { additional anti-VEGF + PRN addi- } \\
\text { tional PDT } \\
\text { 2. Late combination group: PRN addi- } \\
\text { tional Anti-VEGF, and allowed PRN } \\
\text { PDT (postponed PDT PRN) }\end{array}$ & According to real world practice \\
\hline Gomi et al & $\begin{array}{l}\text { Monthly ranbi- } \\
\text { zumab } 0.5 \mathrm{mg} \times 3\end{array}$ & $\begin{array}{l}\text { Standard fluence } 6 \mathrm{mg} / \mathrm{m} 2 \\
689 \text {-nm wavelengths } 600 \mathrm{~mW} / \mathrm{cm} 2 \\
\text { irradiance, } 83 \mathrm{~s}\end{array}$ & $\begin{array}{l}\text { 1. Early combination group: PRN } \\
\text { additional anti-VEGF monthly and } \\
\text { PRN additional PDT every } 3 \text { month } \\
\text { 2. Late combination group: additional } \\
\text { anti-VEGF monthly and allowed PRN } \\
\text { PDT every } 3 \text { month (postponed PDT } \\
\text { PRN) }\end{array}$ & $\begin{array}{l}\text { 1. Anti-VEGF: Decrease BCVA ETDRs } \\
\text { letter }>5 \\
\text { 2. PDT: BCVA }<=0.7 \text { and polypoidal } \\
\text { lesions were seen with subretinal fluid } \\
\text { on the ICGA images }\end{array}$ \\
\hline Koh et al. (2012) & $\begin{array}{l}\text { Monthly ranbi- } \\
\text { zumab } 0.5 \mathrm{mgx} 3\end{array}$ & $\begin{array}{l}\text { Standard fluence } 6 \mathrm{mg} / \mathrm{m} 2 \\
689 \text {-nm wavelengths } 600 \mathrm{~mW} / \mathrm{cm} 2 \\
\text { irradiance, } 83 \mathrm{~s}\end{array}$ & $\begin{array}{l}\text { 1. PDT group: PRN additional PDT } \\
\text { monthly } \\
\text { 2. Anti-VEGF group: PRN additional } \\
\text { anti-VEGF monthly } \\
\text { 3. Early combination group: PRN } \\
\text { addition PDT monthly and PRN } \\
\text { additional anti-VEGF monthly }\end{array}$ & $\begin{array}{l}\text { mainly driven by ICGA-assessed polyp } \\
\text { regression, considering in addition FA } \\
\text { leakage and BCVA }\end{array}$ \\
\hline Koh et al. (2017) & $\begin{array}{l}\text { Monthly ranbi- } \\
\text { zumab 0.5 mgx3 }\end{array}$ & $\begin{array}{l}\text { Standard fluence } 6 \mathrm{mg} / \mathrm{m} 2 \\
689 \text {-nm wavelengths } 600 \mathrm{~mW} / \mathrm{cm} 2 \\
\text { irradiance, } 83 \mathrm{~s}\end{array}$ & $\begin{array}{l}\text { 1. Anti-VEGF group: PRN additional } \\
\text { anti-VEGF monthly } \\
\text { 2. Early combination group: PRN } \\
\text { additional anti-VEGF monthly and } \\
\text { additional PDT every } 3 \text { month }\end{array}$ & $\begin{array}{l}\text { 1. Anti-VEGF: Decrease BCVA or } \\
\text { presence of OCT anomaly } \\
\text { 2. PDT: presence of active PCV (pol- } \\
\text { yps or leakage) on ICGA or FA }\end{array}$ \\
\hline Lai et al & $\begin{array}{l}\text { Ranbizumab } \\
0.5 \text { mgx } 1\end{array}$ & $\begin{array}{l}\text { Standard fluence } 6 \mathrm{mg} / \mathrm{m} 2 \\
689 \text {-nm wavelengths } 600 \mathrm{~mW} / \mathrm{cm} 2 \\
\text { irradiance, } 83 \mathrm{~s}\end{array}$ & $\begin{array}{l}\text { 1. PDT group: PRN additional PDT } \\
\text { every } 3 \text { month } \\
\text { 2. Anti-VEGF group: PRN additional } \\
\text { anti-VEGF monthly } \\
\text { 3. Early combination group: PRN } \\
\text { additional anti-VEGF monthly and } \\
\text { additional PDT every } 3 \text { month }\end{array}$ & $\begin{array}{l}\text { According to PrONTO study } \\
\text { Decrease BCVA ETDRs letter }>5 \text { or } \\
\text { CFT increase }>100 \text { um in OCT orPED } \\
\text { enlargement or macula hemorrhage or } \\
\text { new PCV orpersistent fluid on OCT }\end{array}$ \\
\hline Lee et al & $\begin{array}{l}\text { Monthly afliber- } \\
\text { cept } 2 \mathrm{mg} \times 3 \text { then } \\
2 \text {-monthly in } \\
\text { patient no need } \\
\text { rescue therapy) }\end{array}$ & $\begin{array}{l}\text { According to the current Visudyne } \\
\text { package labeling }\end{array}$ & $\begin{array}{l}\text { 1. Anti-VEGF group: PRN additional } \\
\text { anti-VEGF monthly } \\
\text { 2. Late combination: PRN additional } \\
\text { anti-VEGF monthly and allowed PRN } \\
\text { PDT every } 3 \text { month ( postponed PDT } \\
\text { PRN) }\end{array}$ & $\begin{array}{l}\text { BCVA }<73 \text { letter and }(\text { BCVA gain }<5 \\
\text { ETDRS letter or }>=5 \text { but }<=10 \\
\text { ETDRS and PDT might be beneficial) } \\
\text { and } \\
\begin{array}{l}\text { New or persistent fluid in OCT and } \\
\text { presence of active PCV on ICGA }\end{array}\end{array}$ \\
\hline Li et al & $\begin{array}{l}\text { Ranbizumab } \\
0.5 \mathrm{mlx} 3\end{array}$ & $\begin{array}{l}\text { Reduced fluence } 10.5 \mathrm{mg} \text {, spot size } \\
800 \mathrm{~mm} \text {,Lesion was irradiated for } 70^{\prime \prime} \\
\text { at } 600 \mathrm{~mW} / \mathrm{cm} 2 \text { and } 42 \mathrm{~J} / \mathrm{cm} 2\end{array}$ & $\begin{array}{l}\text { 1. Anti-VEGF group: PRN additional } \\
\text { anti-VEGF monthly } \\
\text { 2. Early combination group: PRN } \\
\text { additional anti-VEGF monthly and } \\
\text { additional PDT every } 3 \text { month }\end{array}$ & $\begin{array}{l}\text { According to PrONTO study } \\
\text { Decrease BCVA ETDRs letter }>5 \text { or } \\
\text { CFT increase }>100 \text { um in OCT orPED } \\
\text { enlargement or macula hemorrhage or } \\
\text { new PCV or persistent fluid on OCT } \\
\text { or active leakage on FAG }\end{array}$ \\
\hline Lim et al. & $\begin{array}{l}\text { Bevacizumab } \\
0.05 \mathrm{ml} \text { every } \\
6 \text { weeks }\end{array}$ & & $\begin{array}{l}\text { 1. Anti-VEGF group: PRN additional } \\
\text { anti-VEGF at 18,24,32,48 weeks } \\
\text { 2. PRN additional anti-VEGF at } \\
\text { 18,24,32,48 weeks } \\
\text { 3. Early combination group: PRN } \\
\text { additional anti-VEGF monthly and } \\
\text { additional PDT }\end{array}$ & $\begin{array}{l}\text { CFT increased by more than } 100 \text { um } \\
\text { New SRF }\end{array}$ \\
\hline Oishi et al & $\begin{array}{l}\text { Monthly } \\
\text { ranbizumab } \\
0.5 \text { mgx3 }\end{array}$ & $\begin{array}{l}\text { Standard fluence } 6 \mathrm{mg} / \mathrm{m} 2689-\mathrm{nm} \\
\text { wavelengths } 600 \mathrm{~mW} / \mathrm{cm} 2 \text { irradiance, } \\
83 \mathrm{~s}\end{array}$ & $\begin{array}{l}\text { 1. Anti-VEGF group: PRN additional } \\
\text { anti-VEGF monthly } \\
\text { 2. PDT group: PRN additional PDT } \\
\text { every } 1.5 \text { month }\end{array}$ & $\begin{array}{l}\text { According to PrONTO study decrease } \\
\text { BCVA ETDRs letter }>5 \text { or CFT } \\
\text { increase }>100 \text { um in OCT orPED } \\
\text { enlargement or macula hemorrhage or } \\
\text { new PCV or persistent fluid on OCT } \\
\text { or active leakage on FAG }\end{array}$ \\
\hline Teo et al. & $\begin{array}{l}\text { Bevacizumab16 } \\
\text { people Aflibercept } \\
10 \text { people }\end{array}$ & N/A & $\begin{array}{l}\text { 1. Anti-VEGF group: PRN additional } \\
\text { anti-VEGF monthly } \\
\text { 2. Early combinatio group: PRN addi- } \\
\text { tional anti-VEGF monthly }\end{array}$ & $\begin{array}{l}\text { if intraretinal or subretinal fluid } \\
\text { persisted }\end{array}$ \\
\hline
\end{tabular}

Table 2. Treatment strategy.

Chong reported results similar to our analysis. By contrast, Chen et al. and Li et al. reported that combination therapy led to significantly greater BCVA change than did anti-VEGF monotherapy.

BCVA improvement rate. Most trials defined BCVA improvement as an EDTR gain of at least $>15$ words. Only Lai et al. set the EDTR gain at this threshold. Of the 7 RCTs, 5 reported this outcome. No significant differences were detected in this network meta-analysis. The SUCRA value revealed that late combination therapy could be 

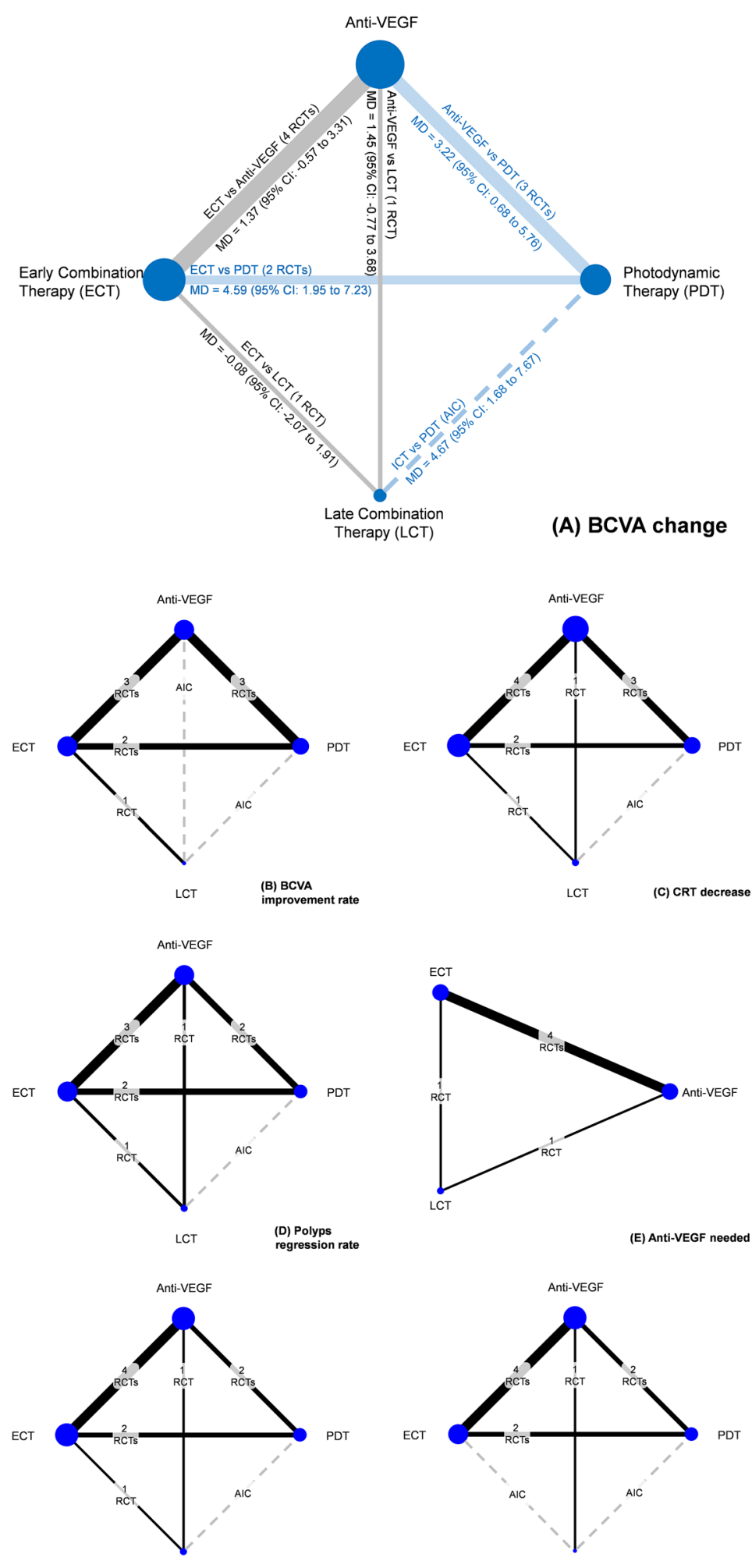

LCT (F) Systemic adverse event

LCT (G) Ocular adverse event

Figure 1. Network plots.

the optimal therapy in terms of BCVA improvement (SUCRA value 69.4). Notably, only one non-RCT reported this outcome. Li et al. suggested that the early combination group had a higher BCVA improvement rate than the anti-VEGF group. 


Anti-VEGF vs PDT
ECT vs PDT
LCT vs PDT
ECT vs Anti-VEGF
LCT vs Anti-VEGF
LCT vs ECT

Figure 2. Forest plot of main findings.

\begin{tabular}{|c|c|c|c|c|c|c|c|c|c|}
\hline \multicolumn{2}{|l|}{ Comparison } & \multirow[t]{2}{*}{ Studies (n) } & \multicolumn{2}{|c|}{ Effect size } & \multirow{2}{*}{\begin{tabular}{|l|}
$I^{2}$ \\
$(\%)$ \\
\end{tabular}} & \multicolumn{2}{|c|}{ Inconsistency } & \multicolumn{2}{|c|}{ Egger test } \\
\hline Arm 1 & Arm 2 & & WMD & $95 \% \mathrm{CI}$ & & $\chi^{2}$ & $P$ & $t$ & $P$ \\
\hline BCVA improvement rate \% & & & & & & 4.28 & 0.12 & -1.93 & 0.10 \\
\hline Anti-VEGF & PDT & 3 & 1.26 & 0.67 to 3.63 & 0 & & & & \\
\hline ECT & PDT & 2 & 1.49 & 0.71 to 3.13 & 0 & & & & \\
\hline LCT & PDT & AIC & 1.61 & 0.55 to 4.68 & & & & & \\
\hline ECT & Anti-VEGF & 3 & 1.18 & 0.64 to 2.16 & 52 & & & & \\
\hline LCT & Anti-VEGF & AIC & 1.27 & 0.48 to 3.38 & & & & & \\
\hline LCT & ECT & 1 & 1.08 & 0.50 to 2.33 & & & & & \\
\hline \multicolumn{6}{|c|}{ Extra anti-VEGF needed (Numbers of injections) } & 3.25 & 0.07 & 0.41 & 0.70 \\
\hline ECT & Anti-VEGF & 4 & -0.72 & -2.07 to 0.63 & 89.2 & & & & \\
\hline LCT & Anti-VEGF & 1 & -1.44 & -3.61 to 0.72 & & & & & \\
\hline LCT & ECT & 1 & -0.72 & -2.02 to 1.48 & & & & & \\
\hline
\end{tabular}

Table 3. Summary of outcomes. AIC, adjusted indirect comparison; Anti-VEGF, anti-vascular endothelial growth factor; BCVA, best-corrected visual acuity; CI, confidence interval; ECT, early combination therapy; LCT, late combination therapy; PDT, photodynamic therapy; WMD, weighted mean difference.

Complete polyp regression rate. Of the 7 RCTs, 5 reported the outcome of complete polyp regression rate $^{7-10,13}$. The anti-VEGF group had a significantly lower complete polyp regression rate than the PDT group (RR: $0.46 ; 95 \%$ CI: $0.28,0.74$ ), and the early combination group had a significantly higher complete polyp regression rate than the anti-VEGF group (RR: 1.91; 95\% CI: 1.40, 2.60). Even though no significant differences were detected between the late and early combination groups, the early combination group exhibited a more favorable trend. Regarding other non-RCTs, 2 of 4 reported this outcome. Li et al. reported no significant differences between the early combination group and anti-VEGF group regarding this outcome. However, Teo et al. reported that the early combination group had a significantly higher complete polyp regression rate than the anti-VEGF group.

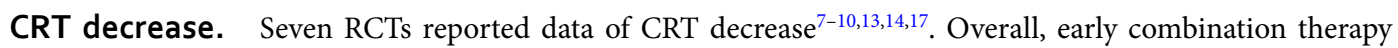
led to a significantly greater decrease in CRT than did PDT (WMD: 54.83; 95\% CI: 12.41, 97.25), anti-VEGF (WMD: 43.40; 95\% CI: 19.84, 66.96), or late combination (WMD:-38.66; 95\% CI: - 51.55, 25.77). No significant differences were noted upon comparison of the late combination group with the PDT and anti-VEGF groups. Therefore, early combination therapy might be the optimal treatment modality for reducing CRT. Notably, 2 non-RCTs presented data of CRT decrease. Li et al. reported that early combination therapy caused a more sig- 
nificant decrease in CRT than did anti-VEGF, which is in agreement with the results of our analysis. By contrast, Teo et al. could not detect a significant difference with the same comparison during a 3-month follow-up.

Total number of anti-VEGF injections. Regarding this outcome, comparisons were only performed among anti-VEGF, early combination, and late combination groups. Overall, 6 of the 7 RCTs reported the total number of anti-VEGF injections during follow-up $p^{7,9,10,13,14,17}$. We could not detect a significant difference in the analysis. High heterogenicity $\left(\mathrm{I}^{2}=89.2 \%\right)$ was noted in the comparative analysis between early combination and anti-VEGF groups. The origin of heterogenicity was the study by Lim et al. We speculated that it could be attributable to the small sample size of their study ( $n=5$ in each group). The SUCRA value revealed that late combination therapy might require fewer anti-VEGF injections (SUCRA value 81.9). All non-RCTs presented the data for this outcome. Chong et al. reported that the early combination group required significantly fewer anti-VEGF than did the late combination group. Li et al. reported a significant difference between early combination and anti-VEGF groups. By contrast, Teo et al. observed only a marginally significant difference $(P=0.05)$ with the same comparison. Chen et al. reported that the combination therapy and anti-VEGF groups required a similar number of anti-VEGF injections.

Safety. Systematic adverse events and Ocular adverse events. Of the 7 RCTs, 6 reported data of systematic adverse events $7,9,10,13,14,17$. Overall, no significant difference was detected in this analysis. Regarding ocular adverse events, Five RCTs reported data on the outcome of ocular adverse events $s^{7,10,13,14,17}$. The network metaanalysis revealed no significant differences related to ocular adverse events among all treatment modalities.

Grading of recommendations, assessment, development, and evaluation. Overall, the certainty of the evidence was low to moderate in our analysis. The level of evidence was downgraded because of the high risk of bias from randomization in most studies as well as indirectness and imprecision. Details are provided in Supplementary 22.

\section{Discussion}

This study is the first systematic review and network meta-analysis of PCV treatments. We included 11 studies published before February 2020 and analyzed 7 RCTs. Notably, the results of the prospective observational studies were heterogenous and not fully compatible with our network meta-analysis of RCTs. Our analysis revealed that early combination therapy might be the optimal therapy in terms of anatomical outcome. However, in terms of BCVA change, anti-VEGF monotherapy was not inferior to the 2 combination therapy modalities.

A significant finding of our study is that the early combination group had the best anatomical outcomes, including complete polyp regression rate and CRT decrease. To our knowledge, anatomical outcomes are the primary focus of PCV treatment; however, the association between polyp closure rate and long-term recurrence rate remains unclear ${ }^{23}$. This finding was different from that of a crucial Fujisan study that reported similar CRT decrease in early and late combination groups ${ }^{9}$. Furthermore, Regarding BCVA change, despite combination therapy exhibiting more favorable trends, our results revealed no significant differences between the 2 modalities of combination therapy and anti-VEGF therapy, which is in agreement with the findings of most studies, $71,14,16-18$. By contrast, EVEREST II reported that the early combination group had a significantly greater BCVA change than did the anti-VEGF group. This heterogenicity could be attributable to the relatively better baseline BCVA in the population of EVEREST $\mathrm{II}^{10}$. Moreover, we noticed that the results of Lim et al. ${ }^{17}$ differed from those of other studies ${ }^{17}$, possibly because of the small sample size $(n=10)$ in their study.

Nevertheless, variations among the population characteristics and treatment protocols merit further exploration and discussion. This information might provide useful insight for clinical practice. We summarized the important information including type of Anti-VEGF agent, protocol of PDT and protocol of rescue therapy in Supplementary 3. Notably, the use of different anti-VEGF agents might be a crucial aspect of PCV treatment. Several studies have reported aflibercept monotherapy to be beneficial in terms of BCVA and anatomical outcome ${ }^{24,25}$. Kawashima et al. reported the effects of aflibercept in ranibizumab-resistant PCV. They indicated a significant difference between BCVA at 6 months and at baseline, albeit with no significant CRT decrease ${ }^{26}$. Recently, Azuma et al. reported the 2-year outcome of treat-and-extend aflibercept for ranibizumab-resistant PCV. They observed a significant CRT decrease from baseline. However, significant BCVA improvement could be observed only at the 1-year mark ${ }^{27}$. Several trials have directly compared the effects of aflibercept and ranibizumab in treating PCV. Notably, even though no significant BCVA change was observed among various antiVEGF agent groups, the aflibercept group exhibited better anatomical outcomes ${ }^{28,29}$. Among our included RCTs, only one study used aflibercept as the anti-VEGF agent ${ }^{14}$. Nonetheless, because of insufficient data, we could not further compare the effects of aflibercept and ranibizumab with meta-regression or subgroup analysis in our network meta-analysis. Hence, further RCTs are warranted to explore efficacy of different type of anti-VEGF monotherapy and combination therapy.

Because of the recurrent nature of PCV, follow-up time is another critical issue in PCV treatment. A previous systematic review reported that the effect of early PDT could be maintained for 2 years. However, the PCV recurrence rate ranged from $40 \%$ to $78.6 \%$ after 3 years $^{23}$. Nonetheless, the long-term outcomes of combination therapy were reported recently. Miyata et al. reported that BCVA improved relative to the baseline only in the first year and not after 3 to 5 years. Moreover, CRT after 5 years was reported to be similar to the baseline CRT ${ }^{30}$. Wataru et al. detected a similar deterioration of BCVA improvement after 3 years. However, a significant BCVA improvement was maintained for 5 years. They speculated that the difference between the 2 trials was attributable to the age of their population ${ }^{31}$. The longest follow-up time included in our prospective study was 2 years. Therefore, our analysis could not evaluate long-term changes. 
Regarding the safety profile, our study revealed no significant differences related to systematic or ocular adverse events among the therapeutic modalities, and few severe adverse events were reported among these studies. However, several reports have indicated that repeated PDT might damage the retinal pigment epithelium and choriocapillaris layer ${ }^{32,33}$. In addition, Miyata et al. reported a marginally significant increase $(P=0.06)$ in rate of macular atrophy in the combination therapy group, and they considered repeated PDT to be related to macular atrophy in the long term ${ }^{30}$. Nonetheless, there is still no sufficient evidence regarding the long-term consequences of PDT.

Limitations. Our study had some limitations. First, because of the limited number of RCTs, only 7 RCTs were included in the network meta-analysis. Meta-regression could not be performed for potential confounding factors. Second, CRT measurements reported in the eligible RCTs covered different ranges. Some of them covered the central subfield ${ }^{10,14}$, whereas others only targeted the central fovea. However, no significant inconsistency or serious heterogeneity existed in the pooled estimate of CRT decrease. Third, the follow-up time in most of our included studies was 1 year; therefore, the long-term effects of PCV therapy require evaluation in future studies. Finally, because of much higher incidence and prevalence of PCV in Asian, it was lack of data for other ethnicity. Further trial in other country is warranted for more comprehensive global perspective.

\section{Conclusions}

This study is the first systematic review and network meta-analysis regarding PCV therapy. We recruited prospective observational trials and RCTs. In addition, we critically appraised these studies and performed quantitative analysis of RCTs. Our results revealed that even though no significant differences related to BCVA change were observed among anti-VEGF, late combination, and early combination groups, combination therapy, especially early combination therapy, could result in better anatomical outcomes. Further trials are warranted to investigate the crucial aspects of PCV therapy, such as long-term effects, cost effectiveness, and predictors of therapy response.

Received: 8 October 2020; Accepted: 19 January 2021

Published online: 02 February 2021

\section{References}

1. Cheung, C. M. G. et al. Polypoidal choroidal vasculopathy: definition, pathogenesis, diagnosis, and management. Ophthalmology 125, 708-724. https://doi.org/10.1016/j.ophtha.2017.11.019 (2018).

2. Brown, D. M. et al. Ranibizumab versus verteporfin for neovascular age-related macular degeneration. N Engl J Med 355, 14321444. https://doi.org/10.1056/NEJMoa062655 (2006).

3. Rosenfeld, P. J. et al. Ranibizumab for neovascular age-related macular degeneration. N Engl J Med 355, 1419-1431. https://doi. org/10.1056/NEJMoa054481 (2006).

4. Nowak-Sliwinska, P., van den Bergh, H., Sickenberg, M. \& Koh, A. H. Photodynamic therapy for polypoidal choroidal vasculopathy. Progress Retinal Eye Res. 37, 182-199. https://doi.org/10.1016/j.preteyeres.2013.09.003 (2013).

5. Otani, A. et al. Indocyanine green angiography: guided photodynamic therapy for polypoidal choroidal vasculopathy. Am. J. Ophthalmol. 144, 7-14. https://doi.org/10.1016/j.ajo.2007.03.014 (2007).

6. Newman, D. K. Photodynamic therapy: current role in the treatment of chorioretinal conditions. Eye 30, 202-210. https://doi. org/10.1038/eye.2015.251 (2016).

7. Koh, A. et al. Everest study: Efficacy and safety of verteporfin photodynamic therapy in combination with ranibizumab or alone versus ranibizumab monotherapy in patients with symptomatic macular polypoidal choroidal vasculopathy. Retina 32, 1453-1464. https://doi.org/10.1097/IAE.0b013e31824f91e8 (2012).

8. Oishi, A. et al. Comparison of the effect of ranibizumab and verteporfin for polypoidal choroidal vasculopathy: 12-month LAPTOP study results. Am. J. Ophthalmol. 156, 644-651. https://doi.org/10.1016/j.ajo.2013.05.024 (2013).

9. $9 \mathrm{Gomi}$, F. et al. Initial versus delayed photodynamic therapy in combination with ranibizumab for treatment of polypoidal choroidal vasculopathy: the Fujisan Study. Retina (philadelphia, pa.) 35, 1569-1576, https://doi.org/10.1097/IAE.0000000000000526 (2015).

10. Koh, A. et al. Efficacy and safety of ranibizumab with or without verteporfin photodynamic therapy for polypoidal choroidal vasculopathy: a randomized clinical trial. JAMA Ophthalmol. 135, 1206-1213. https://doi.org/10.1001/jamaophthalmol.2017.4030 (2017).

11. 11Lai, T. Y. Y., Feller, C., Margaron, P. \& Tan, C. S. Anatomical outcomes of ranibizumab $0.5 \mathrm{mg}$ combined with verteporfin photodynamic therapy vs ranibizumab monotherapy in patients with polypoidal choroidal vasculopathy: 12 -month results from the EVEREST II study. Investigative Ophthalmol. Vis. Sci. 58 (2017).

12. 12 Chen, S.-N. et al. One-year real-world outcomes of ranibizumab $0.5 \mathrm{mg}$ treatment in Taiwanese patients with polypoidal choroidal vasculopathy: a subgroup analysis of the REAL study. Int. J. Ophthalmol. 11, 1802-1808, https://doi.org/10.18240/ijo.2018.11.11 (2018).

13. Lai, K. et al. Comparison of the effects of photodynamic therapy, intravitreal ranibizumab and combination for polypoidal choroidal vasculopathy under $1+$ PRN regimen. BMC Ophthalmol 18, 144-144. https://doi.org/10.1186/s12886-018-0801-7 (2018).

14. Lee, W. K. et al. Efficacy and safety of intravitreal aflibercept for polypoidal choroidal vasculopathy in the planet study: a randomized clinical trial. JAMA Ophthalmol. 136, 786-793. https://doi.org/10.1001/jamaophthalmol.2018.1804 (2018).

15. Li, J., Sun, J., Li, B. \& Liu, Z. Intravitreal ranibizumab injection combined with photodynamic therapy for polypoidal choroidal vasculopathy. Exp. Ther. Med. 15, 1546-1551. https://doi.org/10.3892/etm.2017.5565 (2018).

16. 16Teo, K. Y. C. et al. Comparison of optical coherence tomography angiographic changes after anti-vascular endothelial growth factor therapy alone or in combination with photodynamic therapy in polypoidal choroidal vasculopathy. Retina (Philadelphia, Pa.) 38, 1675-1687, https://doi.org/10.1097/IAE.0000000000001776 (2018).

17. Lim, J. Y. et al. Intravitreal bevacizumab alone versus in combination with photodynamic therapy for the treatment of neovascular maculopathy in patients aged 50 years or older: 1-year results of a prospective clinical study. Acta Ophthalmol. 90, 61-67. https:// doi.org/10.1111/j.1755-3768.2009.01841.x (2012).

18. 18Chong Teo, K. Y. et al. A Multicountry comparison of real-world management and outcomes of polypoidal choroidal vasculopathy: fight retinal blindness! cohort. Ophthalmol Retina 3, 220-229, https://doi.org/10.1016/j.oret.2018.11.003 (2019). 
19. Wang, W., He, M. \& Zhang, X. Combined intravitreal anti-VEGF and photodynamic therapy versus photodynamic monotherapy for polypoidal choroidal vasculopathy: a systematic review and meta-analysis of comparative studies. PLOS ONE 9, e110667-e110667. https://doi.org/10.1371/journal.pone.0110667 (2014).

20. Tang, K. et al. Ranibizumab alone or in combination with photodynamic therapy vs photodynamic therapy for polypoidal choroidal vasculopathy: a systematic review and Meta-analysis. Int. J. Ophthalmol. 8, 1056-1066. https://doi.org/10.3980/j.issn.22223959.2015.05.36 (2015).

21. Yong, M., Zhou, M. \& Deng, G. Photodynamic therapy versus anti-vascular endothelial growth factor agents for polypoidal choroidal vasculopathy: a meta-analysis. BMC Ophthalmol. 15, 82-82. https://doi.org/10.1186/s12886-015-0064-5 (2015).

22. Qian, T., Li, X., Zhao, M. \& Xu, X. Polypoidal choroidal vasculopathy treatment options: A meta-analysis. Eur. J. Clin. Invest. 48, e12840. https://doi.org/10.1111/eci.12840 (2018).

23. Wong, C. W. et al. THREE-year results of polypoidal choroidal vasculopathy treated with photodynamic therapy: retrospective study and systematic review. RETINA 35, 1577-1593. https://doi.org/10.1097/iae.0000000000000499 (2015).

24. Arakawa, A., Inoue, M., Sato, S., Yamane, S. \& Kadonosono, K. Efficacy of intravitreal aflibercept injections for Japanese patients with polypoidal choroidal vasculopathy. Clin. Ophthalmol. 11, 797-802. https://doi.org/10.2147/OPTH.S129164 (2017).

25. Lee, J. E. et al. Efficacy of fixed-dosing aflibercept for treating polypoidal choroidal vasculopathy: 1-year results of the VAULT study. Graefes Arch. Clin. Exp. Ophthalmol. 255, 493-502. https://doi.org/10.1007/s00417-016-3489-5 (2017).

26. Kawashima, Y. et al. Effects of aflibercept for ranibizumab-resistant neovascular age-related macular degeneration and polypoidal choroidal vasculopathy. Graefes Arch. Clin. Exp. Ophthalmol. 253, 1471-1477. https://doi.org/10.1007/s00417-014-2838-5 (2015).

27. Azuma, K. et al. Two-year outcome of treat-and-extend aflibercept after ranibizumab in age-related macular degeneration and polypoidal choroidal vasculopathy patients. Clin. Ophthalmol. 12, 1589-1597. https://doi.org/10.2147/OPTH.S172115 (2018).

28. Cho, H. J. et al. Intravitreal aflibercept and ranibizumab injections for polypoidal choroidal vasculopathy. Am. J. Ophthalmol. 165, 1-6. https://doi.org/10.1016/j.ajo.2016.02.019 (2016).

29. Jung, B. J. et al. Intravitreal aflibercept and ranibizumab for pachychoroid neovasculopathy. Sci. Rep. 9, 2055. https://doi. org/10.1038/s41598-019-38504-y (2019).

30. Miyata, M. et al. Five-year visual outcomes after anti-VEGF therapy with or without photodynamic therapy for polypoidal choroidal vasculopathy. Br. J. Ophthalmol. 103, 617. https://doi.org/10.1136/bjophthalmol-2018-311963 (2019).

31. Wataru, K. et al. Five-year outcomes of photodynamic therapy combined with intravitreal injection of ranibizumab or aflibercept for polypoidal choroidal vasculopathy. PLoS ONE 15, e0229231. https://doi.org/10.1371/journal.pone.0229231 (2020).

32. 32Schmidt-Erfurth, U., Laqua, H., Schlötzer-Schrehard, U., Viestenz, A. \& Naumann, G. O. Histopathological changes following photodynamic therapy in human eyes. Arch. Ophthalmol. (Chicago, Ill. : 1960) 120, 835-844 (2002).

33. Mitchell, P. et al. Intravitreal aflibercept (IVT-AFL) with and without photodynamic therapy (PDT) in polypoidal choroidal vasculopathy (PCV): anatomical outcomes assessed by optical coherence tomography (OCT) in the planet study. Clin. Exp. Ophthalmol. 45, 31-32. https://doi.org/10.1111/ceo.13049/full (2017).

\section{Acknowledgements}

We would like to thank Wallace Academic Editing for editing and proofreading this manuscript.

\section{Author contributions}

SCC designed the study. SCC and YNK did the literature search, data extraction, quality assessment of the included studies and data analysis. SCC and YNK wrote the first draft of the study. HYM supervised and revised the manuscript.

\section{Funding}

No funding or support from any government and non-government organization.

\section{Competing interests}

The authors declare no competing interests

\section{Additional information}

Supplementary Information The online version contains supplementary material available at https://doi. org/10.1038/s41598-021-82316-y.

Correspondence and requests for materials should be addressed to Y.-M.H.

Reprints and permissions information is available at www.nature.com/reprints.

Publisher's note Springer Nature remains neutral with regard to jurisdictional claims in published maps and institutional affiliations.

Open Access This article is licensed under a Creative Commons Attribution 4.0 International License, which permits use, sharing, adaptation, distribution and reproduction in any medium or format, as long as you give appropriate credit to the original author(s) and the source, provide a link to the Creative Commons licence, and indicate if changes were made. The images or other third party material in this article are included in the article's Creative Commons licence, unless indicated otherwise in a credit line to the material. If material is not included in the article's Creative Commons licence and your intended use is not permitted by statutory regulation or exceeds the permitted use, you will need to obtain permission directly from the copyright holder. To view a copy of this licence, visit http://creativecommons.org/licenses/by/4.0/.

(C) The Author(s) 2021 